\title{
A Novel Active Anomaly Discovery Method and its Applications in Additive Manufacturing
}

This paper was downloaded from TechRxiv (https://www.techrxiv.org).

LICENSE

CC BY 4.0

SUBMISSION DATE / POSTED DATE

24-09-2021 / 29-09-2021

\section{CITATION}

Shen, Bo; Kong, Zhenyu (2021): A Novel Active Anomaly Discovery Method and its Applications in Additive Manufacturing. TechRxiv. Preprint. https://doi.org/10.36227/techrxiv.16674412.v1

DOI

10.36227/techrxiv.16674412.v1 


\title{
A Novel Active Anomaly Discovery Method and its Applications in Additive Manufacturing
}

\author{
Bo Shen ${ }^{\mathrm{a}}$ and Zhenyu (James) Kong ${ }^{\mathrm{a}^{*}}$ \\ ${ }^{a}$ Grado Department of Industrial and Systems Engineering, Virginia Tech, Blacksburg, US \\ ${ }^{*}$ Corresponding Author
}

\begin{abstract}
Anomaly detection aims to identify the true anomalies from a given set of data instances. Unsupervised anomaly detection algorithms are applied to an unlabeled dataset by producing a ranked list based on anomaly scores. Unfortunately, due to the inherent limitations, many of the top-ranked instances by unsupervised algorithms are not anomalies or not interesting from an application perspective, which leads to high false-positive rates. Active anomaly discovery (AAD) is proposed to overcome this deficiency, which sequentially selects instances to get the labeling information and incorporate it into the anomaly detection algorithm to improve the detection accuracy iteratively. However, labeling is often costly. Therefore, the way to balance detection accuracy and labeling cost is essential. Along this line, this paper proposes a novel AAD method to achieve the goal. Our approach is based on the state-of-the-art unsupervised anomaly detection algorithm, namely, Isolation Forest, to extract features. Thereafter, the sparsity of the extracted features is utilized to improve its anomaly detection performance. To enforce the sparsity of the features and subsequent improvement of the detection analysis, a new algorithm based on online gradient descent, namely, Sparse Approximated Linear Anomaly Discovery (SALAD), is proposed with its theoretical Regret analysis. Extensive experiments on both open-source and additive manufacturing datasets demonstrate that the proposed algorithm significantly outperforms the state-of-the-art algorithms for anomaly detection.
\end{abstract}

Keywords: Isolation Forest; Sparsity; Active Anomaly Discovery; Measurement Feedback; Online Gradient Descent; Additive Manufacturing.

\section{Introduction}

Anomaly detection has been an active research area for several decades and plays increasingly important roles in various applications, for example, process monitoring in additive manufacturing Shen et al. (2021a,b), cyber-attack in smart manufacturing Ten et al. (2011), and healthcare analytics in IoT Ukil et al. (2016). The goal of anomaly detection is to identify anomalies, which have patterns in data that do not conform to a well-defined notion of normal behavior Chandola et al. (2009). An anomaly is a data instance generated by a process different from generating the "normal" data instances, referred to as nominal instances. 
In real-world applications, a dataset usually contains a small percentage of anomalies due to the nature of anomalies Das et al. (2020). Therefore, a manual search for anomalies through all instances is not practical.

As a viable solution, unsupervised anomaly detection algorithms ${ }^{1}$ (e.g., Breunig et al. (2000); Knorr and Ng (1998); Liu et al. (2008)) can help address this problem to some extent. The unsupervised anomaly detection algorithms work on an unlabeled dataset directly to provide an anomaly score list where the anomalies have a higher rank than the nominal data. The top-scored instances are identified as candidate anomalies. Since labeling is not needed for the algorithm itself, the time and cost-saving is the most appealing aspect of this solution. However, unsupervised anomaly detection algorithms generally do not perform well due to their deficiency of the high false-positive rate, which significantly constrains their broad applications.

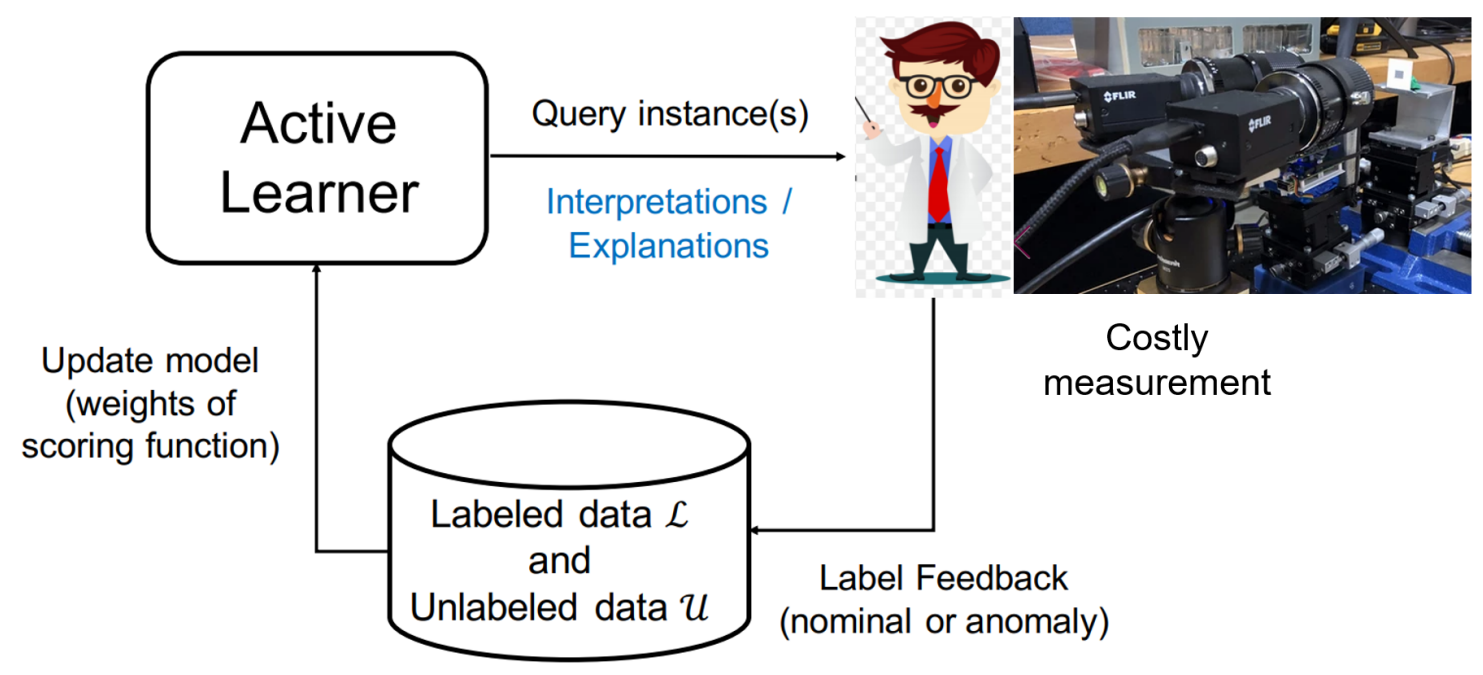

Figure 1: High-level overview of the active anomaly discovery framework Das et al. (2019).

The high false-positive rate of the unsupervised anomaly detection motivates a new research direction, namely, active anomaly discovery (AAD) Das et al. (2017); Siddiqui et al. (2018); Das et al. (2019, 2020). AAD elaborately selects some data instances to label and then utilizes the labeling information to improve the anomaly detection performance. Labeling is obtained through inspections or measurements, which incurs the cost and is

\footnotetext{
${ }^{1}$ The definition of unsupervised anomaly detection is different from works using autoencoder An and Cho (2015), where the autoencoder is trained on all nominal instances
} 
thus needed to be minimized or under a certain constraint. As shown in Figure 1, the goal of $\mathrm{AAD}$ is to sequentially select some data instances to measure and then label (termed "query" in this paper), and maximize the number of detected anomalies under the given number of measurements constrained by the budget.

Among all AAD methods, IF-AAD Das et al. (2017) and Generalized Linear Anomaly Detector (GLAD) Siddiqui et al. (2018) are the state-of-the-art methods. Both apply Isolation Forest Liu et al. (2008) as a feature extraction method to learn a feature function $\phi(\cdot)$, which maps the data instances to high dimensional binary features. At each step, the IF-AAD approach Das et al. (2017) solves an optimization problem based on all prior measurement feedback, which results in new weights for the components. The optimization in this approach involves a number of components associated with hyperparameters. In addition, this optimization problem is non-convex and requires a relatively complex "alternating" convex optimization approach with its own hyperparameters. Both hyperparameters should be optimized for a good performance of IF-AAD, leading to another challenging problem that needs to be solved. This issue makes IF-AAD difficult to be applied in practice.

Rather, this motivates the GLAD method proposed in Siddiqui et al. (2018), which uses a simpler and more efficient approach with a minimal number of parameters. Specifically, they formulated the AAD problem within the framework of online convex optimization. At each step of GLAD: 1) the model weight is updated through online mirror descent Hazan (2019), and 2) Select an instance with the highest anomaly score from all unlabeled instances based on the new weight for investigation. This step yields an efficient and relatively simple approach to incorporating feedback compared to the prior state-of-the-art Das et al. (2017). GLAD has been proven to be one of the best anomaly detection algorithms for large-scale datasets $^{2}$ based on Das et al. (2019) ${ }^{3}$ and package Zhao et al. (2019).

Although Siddiqui et al. (2018) pointed out that the set of features from $\phi(\cdot)$ is very sparse for any particular instance, they did not explore the sparsity further. In this paper, the feature function $\phi(\cdot)$ is proven to be sparse theoretically and verified empirically. We then hypothesized that utilizing the sparsity of extracted features could improve the GLAD

\footnotetext{
${ }^{2}$ The conclusion is drawn from the following link: https://towardsdatascience.com/isolation-forest-is-thebest-anomaly-detection-algorithm-for-big-data-right-now-e1a18ec0f94f

${ }^{3}$ see performance comparison in Appendix B of Das et al. (2019)
} 
algorithm since it can make the anomaly detection algorithm more focused on the essential features. Thus, the objective of this work is to utilize the sparsity of the extracted features in the Isolation Forest-based AAD framework. A new algorithm based on gradient descent, namely, Sparse Approximated Linear Anomaly Discovery (SALAD), is proposed in this paper. The sparsity is achieved by a sparse approximated solution after the gradient descent update at each online round. The key of SALAD is to control the error between the exact solution and the approximated one, which captures the tradeoff between the sparsity of the model and the regret performance of the algorithm.

This paper aims to solve two practical problems in additive manufacturing (AM) using our proposed SALAD: (1) detect a specific type of anomalies from an unlabeled dataset, and (2) measurement allocation under a certain budget. In Sections 4.3 and 4.4, polymer Liu et al. (2019) and metal AM Wang et al. (2021) are used to demonstrate the superior performance of the proposed SALAD in solving Problems (1) and (2), respectively.

The remainder of this paper is organized as follows. The research framework of this paper is provided in Section 2. The isolation forest and sparsity analysis of feature function are presented in Section 3.1, followed by the proposed Sparse Approximated Linear Anomaly Discovery in Section 3.2. The numerical and real-world case studies are provided in Section 4 to test and validate the proposed algorithm. Finally, the conclusions and future work are discussed in Section 5.

\section{Research Framework}

In the setting of this paper, $m$ data instances $\mathbf{X}=\left\{\boldsymbol{x}_{1}, \ldots, \boldsymbol{x}_{m}\right\}$ are considered as a dataset. $\boldsymbol{x}_{i} \in \mathbb{R}^{d}$ is a data instance that is not given with any labels initially. But, it can be queried through measurement with a certain cost and then labeled as either anomaly or nominal class, namely, $y_{i}$ belonging to $\{-1,+1\}$. A query is defined as the procedure to select the data instance and take a measurement for labeling. Instances labeled with +1 represent the anomaly class $\mathbf{A}$. The label of -1 represents the nominal class $\mathbf{N}$. Generally, the nominal instances account for an overwhelming fraction of the data, that is, $|\mathbf{N}| \gg|\mathbf{A}|$. Besides, $\mathcal{L}$ and $\mathcal{U}$ denote the set of labeled and unlabeled instances, respectively.

Our objective is to develop a sampling procedure to sequentially query the unlabeled data instances, which maximizes the number of anomalies to be detected during the query- 
Step 1: Feature Extraction I-ー-ー-ー-ー-

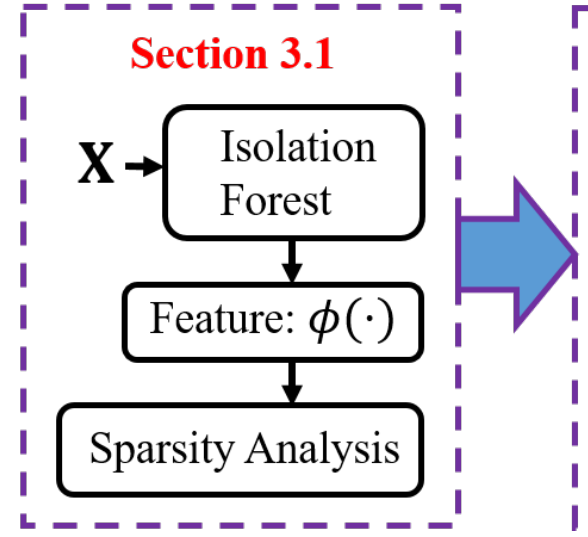

Step 2: Proposed SALAD in Section 3.2 г-ー- - - - - - - - - - - - - 7

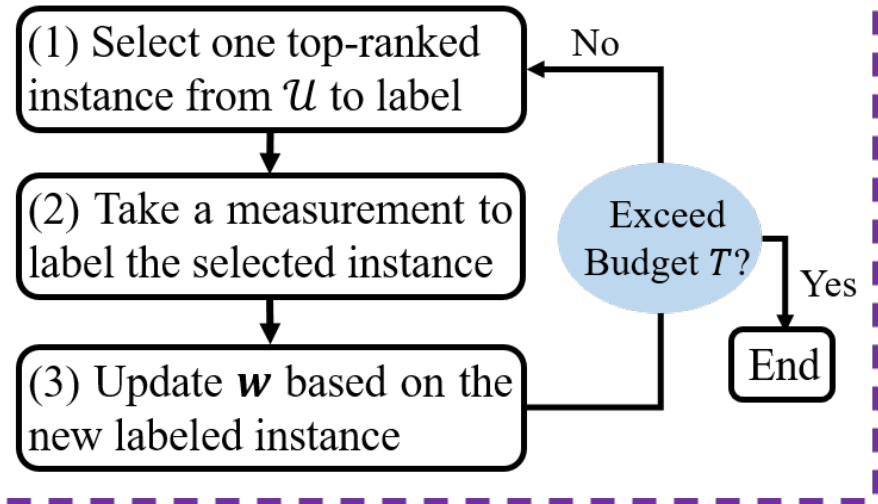

Figure 2: Overview of the framework of the proposed method in this paper.

ing procedure (i.e., let the set of labeled instances $\mathcal{L}$ contains as many anomalies as possible) under a given amount of budget (i.e., a fixed number of measurements). Figure 2 summarizes the framework of our method, which is a two-step procedure. The output from this procedure is the measurements that need to be taken to maximize the probability of capturing anomalies.

- Step 1 (Section 3.1): train Isolation Forest on data $\mathbf{X}$ (for example, the 2D images from the metal AM application in Section 4.4) to obtain its feature function $\phi(\cdot)$. The feature function $\phi(\cdot)$ can be used to calculate the anomaly score for any single instance.

- The anomaly score is utilized to identify top candidates for queries by taking measurements since anomalies are supposed to have high anomaly scores.

- The foundation of Step 2 is the sparsity of feature function $\phi(\cdot)$, which is proved in Section 3.1.2.

- Step 2 (Section 3.2): A new measurement is executed to provide the label for the queried instance (for example, 3D scanner data from the metal AM application). Then the model weight $\boldsymbol{w}$ is updated through the proposed SALAD in Section 3.2 with a sparsity constraint. Specifically, the following loop proceeds until the budget (the maximum number of measurements) is reached: 
(1) Based on the current anomaly scores calculated from model weight $\boldsymbol{w}$, select one top-ranked instance from the unlabeled dataset $\mathcal{U}$;

(2) A new measurement is taken on the queried instance (i.e., provide the label);

(3) Update the model weight $\boldsymbol{w}$ based on the new labeled instance, where $\boldsymbol{w}$ is constrained to be sparse.

The sparsity analysis of feature function $\phi(\cdot)$ motivates our idea to enforce a sparse model weight as summarized in Step 2, making the algorithm focus on more essential features related to anomaly detection.

\section{Proposed Method}

As illustrated in Figure 2, the feature extraction method in Step 1, namely, the Isolation Forest, is introduced with its related theory in Section 3.1. The proposed SALAD in Step 2 is presented in detail in Section 3.2.

\subsection{Isolation Forest and its Sparsity Analysis}

To begin with, feature function $\phi(\cdot)$ and anomaly score from Isolation Forest are introduced in Section 3.1.1. The sparsity of feature function $\phi(\cdot)$ is further analyzed in Section 3.1.2, which is used to develop the algorithm illustrated in Step 2 of Figure 2.

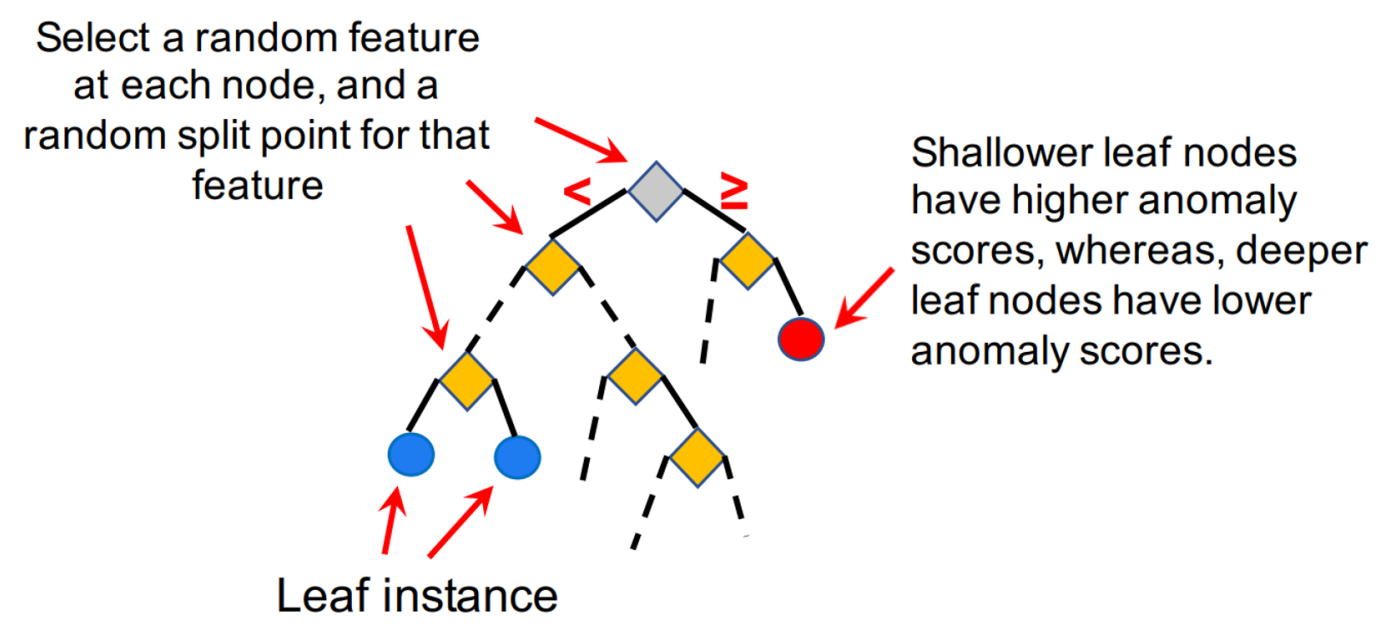

Figure 3: Illustration of one isolation tree from Das et al. (2017). 


\subsubsection{Isolation Forest and its Anomaly Score}

Isolation Forest proposed by Liu et al. $(2008,2012)$ comprises a set of $K$ trees denoted by $\mathbf{T}=\left\{T_{1}, \ldots, T_{K}\right\}$. It is constructed in a randomized manner as illustrated in Figure 3, and outlined in Algorithm 1. Each tree $T_{k}$ is built from the root to leaves by randomly

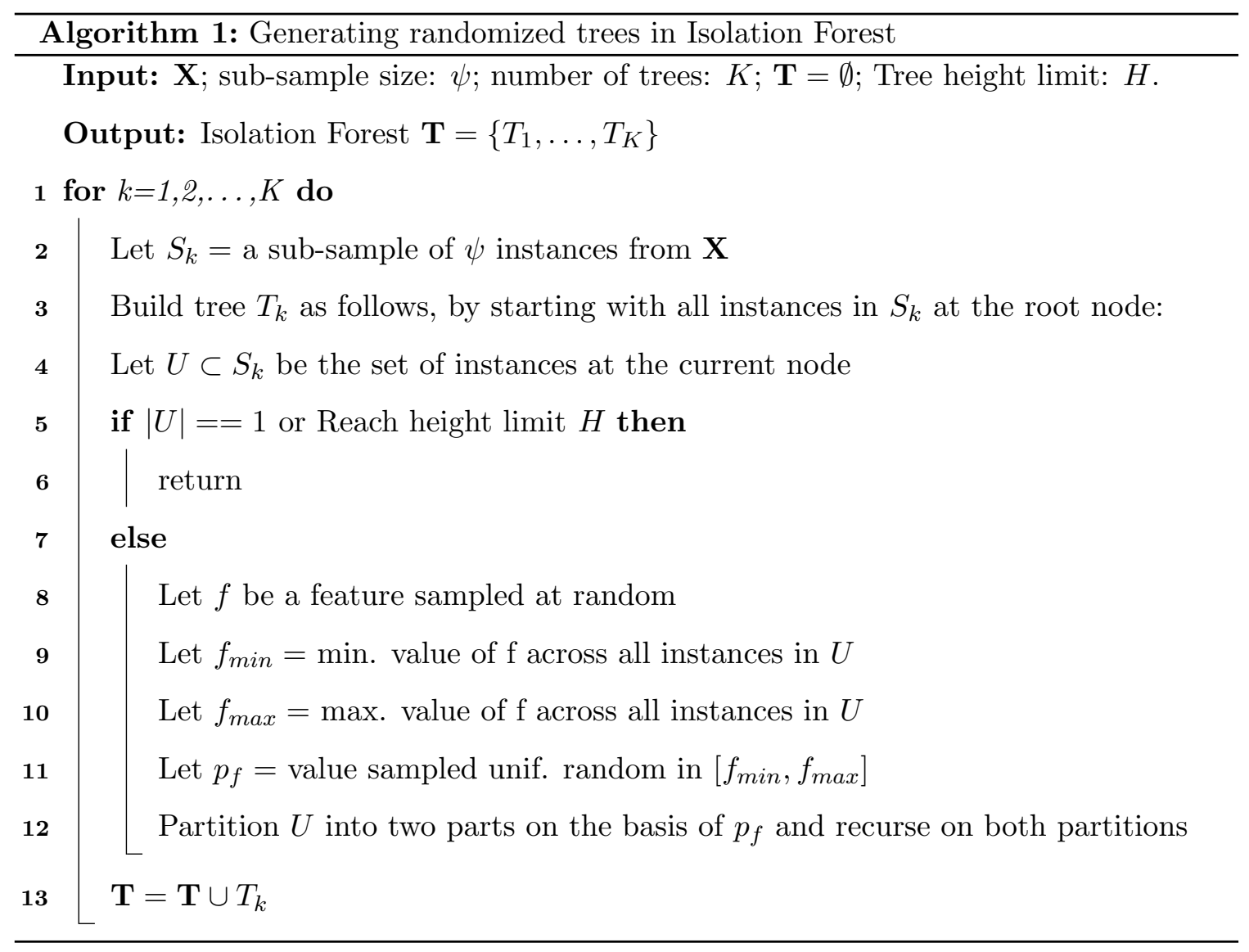

partitioning the data instance at each node via selecting a feature and a threshold both at uniformly random. The trees are grown until each instance is isolated in a leaf or reaches the height limit $H$ (represented as a blue circle in Figure 3). Isolation Forest is based on the idea that anomaly instances are few and well-separated from clusters of nominal instances in the feature space. Because of this, anomaly instances (represented as a red circle in Figure 3) very quickly reach leaf nodes through random partitioning. On the other hand, nominal instances, which form dense clusters, require many more steps to reach leaf nodes finally. Therefore, the length of the path traversed by an instance from the root node to the leaf, also known as the isolation depth, is shorter (on average) for anomaly instances than 
for nominal instances. The anomaly score assigned to an instance is simply the average isolation depth across the forest.

It is straightforward now to describe Isolation Forest as a particular way of setting the weights of a tree-based anomaly detector through a feature function $\phi(\cdot): \mathbb{R}^{d} \rightarrow\{0,1\}^{D}$, where $D$ is the number of edges in the forest. Specifically, the feature function $\phi(\cdot)$ can be constructed as follows: for each edge $e$ in one of the trees,

$$
\phi_{e}(\boldsymbol{x})=\left\{\begin{array}{l}
1, \text { if instance } \boldsymbol{x} \text { goes through the edge } e \\
0, \text { otherwise. }
\end{array}\right.
$$

By doing so, $\phi(\boldsymbol{x})$ is a very sparse feature vector where most features are zero given that each instance can only travel a small number of edges. Similarly, a weight $\boldsymbol{w}_{e}$ is assigned to each edge to construct an anomaly score. Now let $\phi$ and $\boldsymbol{w}$ be vectors that concatenate all of the features and weights across the forest consistently for different instances, and $\boldsymbol{w}_{e}=1$ initially for all $e$. The linear anomaly score function

$$
\operatorname{score}(\boldsymbol{x}, \boldsymbol{w})=-\phi(\boldsymbol{x}) \cdot \boldsymbol{w}
$$

corresponds exactly to the (unnormalized) Isolation Forest anomaly score. The main purpose of making scores negative is to ensure that higher scores indicate more anomalous and lower scores indicate more nominal.

\subsubsection{Sparsity Analysis of Feature Function $\phi(\cdot)$}

This subsection discusses and proves the sparsity of feature function $\phi(\cdot)$ from the Isolation Forest. Once the dataset $\mathbf{X}$ is trained using Algorithm 1, the feature function $\phi: \mathbb{R}^{d} \rightarrow$ $\{0,1\}^{D}$ can be obtained through the process described in (1). The dimension of the binary feature, namely, $D$, is closely related to the tree height limit $H$. Typically, $D$ is increased as increased $H$. The following lemma proves that the binary features from the Isolation Forest feature function $\phi(\cdot)$ are indeed sparse under two conditions.

Lemma 1. Assume that there is no empty leaf in the forest trained by Algorithm 1 on $\psi$ instances, where there are $K$ trees in the forest and $\phi(\cdot)$ is constructed through (1).

(i) If the tree height limit $H$ is small (i.e., not all instances are isolated in a leaf), then for any $\boldsymbol{x} \in \mathbf{X}$

$$
\|\phi(\boldsymbol{x})\|_{0} \leq H \cdot K
$$


(ii) If the tree height limit $H$ is large (i.e., all instances are isolated in a leaf) and $\psi=m$, then

$$
\frac{\lfloor\log m\rfloor}{2(m-1)} \leq r \leq \frac{m+2}{4 m}
$$

where $m$ is the total number of instances in the dataset, $r=\frac{\sum_{i=1}^{m}\left\|\phi\left(\boldsymbol{x}_{i}\right)\right\|_{0}}{m D}$ denotes the ratio of nonzero elements that is calculated from the extracted feature set $\left\{\phi\left(\boldsymbol{x}_{1}\right), \ldots, \phi\left(\boldsymbol{x}_{m}\right)\right\}$, and $D=2(m-1) K$.

Proof. Under Condition ( $i$ ): If the tree height limit is set to $H$, then the number of edges that each instance can travel in each tree is upper bounded by $H$. Since there are $K$ trees in the forest, then $\|\phi(\boldsymbol{x})\|_{0} \leq H \cdot K$.

Under Condition (ii): The dimension of feature function $\phi(\cdot)$, namely, $D$, is first considered. In the case where all instances are isolated in a leaf in a tree, the number of edges in each tree increases by 2 when a new instance is added to the dataset. When $m=2$, $D=2 K$. Therefore, the dimension of feature function $D$ is $\phi(\cdot)$ is $2(m-1) K$.

The upper bound in (4) is proved by induction. When $m=2$, the $\sum_{i=1}^{m}\left\|\phi\left(\boldsymbol{x}_{i}\right)\right\|_{0}=(1+$ 1) $K$; When $m=3$, the $\sum_{i=1}^{m}\left\|\phi\left(\boldsymbol{x}_{i}\right)\right\|_{0}=(1+2+2) K$; When $m=4$, the $\sum_{i=1}^{m}\left\|\phi\left(\boldsymbol{x}_{i}\right)\right\|_{0} \leq$ $(1+2+3+3) K$, where "=" is achieved when the new instance will increase the tree depth by 1 when it is added to the dataset. Therefore, $\sum_{i=1}^{m}\left\|\phi\left(\boldsymbol{x}_{i}\right)\right\|_{0} \leq \frac{(m+2)(m-1) K}{2}$ is valid, when the size of dataset $m \leq 4$. If a new instance is added to the dataset with size $m$ (i.e., it becomes $m+1$ ), the maximum number of edges that the new instance can travel is $m$. Therefore,

$$
\begin{aligned}
\left\|\phi\left(\boldsymbol{x}_{i}\right)\right\|_{0} & \leq \frac{(m+2)(m-1) K}{2}+(1+m) K \\
& =\frac{(m+3) m K}{2},
\end{aligned}
$$

where the second term on the right-hand side of the first inequality comes from the effect of adding one instance. By induction, $\sum_{i=1}^{m}\left\|\phi\left(\boldsymbol{x}_{i}\right)\right\|_{0} \leq \frac{(m+2)(m-1) K}{2}$ is valid for all $m \geq 2$. Thus,

$$
\begin{aligned}
r & \leq \frac{(m+2)(m-1) K}{2 m D} \\
& =\frac{m+2}{4 m} .
\end{aligned}
$$

The lower bound in (4) is also proved by induction. $\sum_{i=1}^{m}\left\|\phi\left(\boldsymbol{x}_{i}\right)\right\|_{0} \geq\lfloor\log m\rfloor m K$ is valid when $m \leq 3$. If a new instance is added the dataset with size $m$ where $2^{\lfloor\log m\rfloor} \leq$ $m \leq 2^{\lceil\log m\rceil}-1$, the minimum number of edges that the new instance can travel is $\lceil\log m\rceil$ 
because all instances at least travel $\lfloor\log m\rfloor$ edges (this is the case where the lower bound is tight when the dataset size is $2^{\lfloor\log m\rfloor}$ ). Therefore,

$$
\begin{aligned}
\sum_{i=1}^{m+1}\left\|\phi\left(\boldsymbol{x}_{i}\right)\right\|_{0} & \geq\lfloor\log m\rfloor m K+\lceil\log m\rceil \\
& =\lfloor\log (m+1)\rfloor(m+1) K
\end{aligned}
$$

By induction, $\sum_{i=1}^{m}\left\|\phi\left(\boldsymbol{x}_{i}\right)\right\|_{0} \geq\lfloor\log m\rfloor m K$ is valid for all $m \geq 2$. As a result,

$$
\begin{aligned}
r & \geq \frac{\lfloor\log m\rfloor m K}{m D} \\
& =\frac{\lfloor\log m\rfloor}{2(m-1)} .
\end{aligned}
$$

Note that the empty leaf only shows up when there are duplicated instances in dataset $\mathbf{X}$ since these instances can not be split in a tree. However, the duplicated instances can be removed so that the assumption of no empty leaf is satisfied. The above lemma discusses the sparsity of feature function $\phi(\cdot)$ under two independent conditions. Conditions (i) and (ii) correspond to the cases of small and large tree height limits, respectively. Under Condition (i), (3) shows the sparsity of $\phi(\boldsymbol{x})$ from any instance is upper bounded by $H \cdot K$. Under Condition (ii), all instances are isolated in a leaf. The inequality (4) shows the lower and upper bound of the ratio of nonzero elements, namely, $r$, that is calculated from the extracted feature set $\left\{\phi\left(\boldsymbol{x}_{1}\right), \ldots, \phi\left(\boldsymbol{x}_{m}\right)\right\}$. As the dataset size $m$ goes to infinity, the lower bound converges to zero while the upper bound converges to 1/4. Lemma 1 characterizes the sparsity of feature function $\phi(\cdot)$, which shows the motivation to include the sparsity constraint with $\phi(\cdot)$.

\subsection{Sparse Approximated Linear Anomaly Discovery}

In this subsection, Step 2 in Figure 2 is presented within the framework of online optimization Shalev-Shwartz et al. (2011a).

\subsubsection{Connection with Online Optimization}

In our problem, the anomaly score in (2) is assigned to an instance $\boldsymbol{x}$, where larger scores correspond to more anomalous instances since anomalous instances are shallower leaf nodes (i.e., there are fewer nonzeros elements in $\phi(\boldsymbol{x})$ for an anomalous instance $\boldsymbol{x})$. At each 
iteration $t$, an anomaly score list over the unlabeled instances can be obtained through $\boldsymbol{w}_{t}$ (Step 2(1) in Figure 2). Based on the anomaly score list, the top-ranked instance is selected for query (i.e., taking measurement/experiment to label the queried instance), which consumes time or cost. After that, measurement feedback $y_{t}$ on the top-ranked instance is received (Step 2(2) in Figure 2), where $y_{t}=+1$ if the instance is an anomaly and $y_{t}=-1$ if it is nominal. To transfer the measurement feedback $y_{t}$ to a function for the online optimization framework, Linear loss is used in this paper due to its efficiency and effectiveness Siddiqui et al. (2018). The linear loss is given by

$$
f_{t}\left(\boldsymbol{w}_{t}\right)=-y_{t} \phi(\boldsymbol{x}) \cdot \boldsymbol{w}_{t}
$$

which is a linear function of $\boldsymbol{w}_{t}$. Intuitively, if $y_{t}=+1$, then our current cost vector should suffer a smaller loss than the case when $y_{t}=-1$, since the ranking based on $\boldsymbol{w}_{t}$ correctly put an anomaly at the top. When anomaly or nominal feedback is received, the model weight $\boldsymbol{w}_{t+1}$ is updated accordingly (Step 2(3) in Figure 2).

The procedure in Step 2 of Figure 2 falls under the framework of online optimization. Online optimization is an iterative game against a potentially an adversary where our moves are vectors from some constrained sets $\mathcal{S}$. The game proceeds as follows: (1) The adversary selects a function $f_{t}: \mathcal{S} \rightarrow \mathbb{R} ;(2)$ Suffer a loss $f_{t}\left(\boldsymbol{w}_{t}\right) ;(3)$ Select a vector $\boldsymbol{w}_{t+1} \in \mathcal{S}$. In our problem, the adversary is the greedy policy to select the top-ranked instance in Step 2(1). Typically, the performance of online optimization is usually measured by the accumulated Regret for the best $\boldsymbol{w}^{*}$ that is the minimizer by assuming $f_{t}$ 's are known in advance. Specifically, the accumulated Regret for a $T$-round game is defined as

$$
\text { Regret }_{T}=\sum_{t=1}^{T} f_{t}\left(\boldsymbol{w}_{t}\right)-\min _{\boldsymbol{w} \in \mathcal{S}} \sum_{t=1}^{T} f_{t}(\boldsymbol{w}) .
$$

The objective of our problem is to minimize the accumulated Regret by choosing the appropriate $\boldsymbol{w}_{t}(t=1, \ldots, T)$ sequentially.

\subsubsection{Sparse Approximated Linear Anomaly Discovery Algorithm}

To minimize (6), a wide variety of online optimization algorithms are available to use in the literature. In particular, online gradient descent techniques Kivinen et al. (2004); Zhang (2004); Shalev-Shwartz et al. (2011b) stand out as an attractive combination of predictive 
accuracy and computational efficiency. Furthermore, it is necessary to add sparsity constraint in model weight $\boldsymbol{w}$ as discussed in Section 3.1. In the literature, there are three main techniques to achieve sparsity: (i) imposing an $\ell_{1}$-norm constraint Duchi et al. (2008); (ii) adding an $\ell_{1}$-norm regularization to the loss function Langford et al. (2009); or (iii) using $\ell_{0}$-based truncation Wang et al. (2013). Our proposed sparsity strategy differs from the above-mentioned approaches. The proposed algorithm, namely, SALAD, is motivated by Zhai et al. (2018) and presented in Algorithm 2.

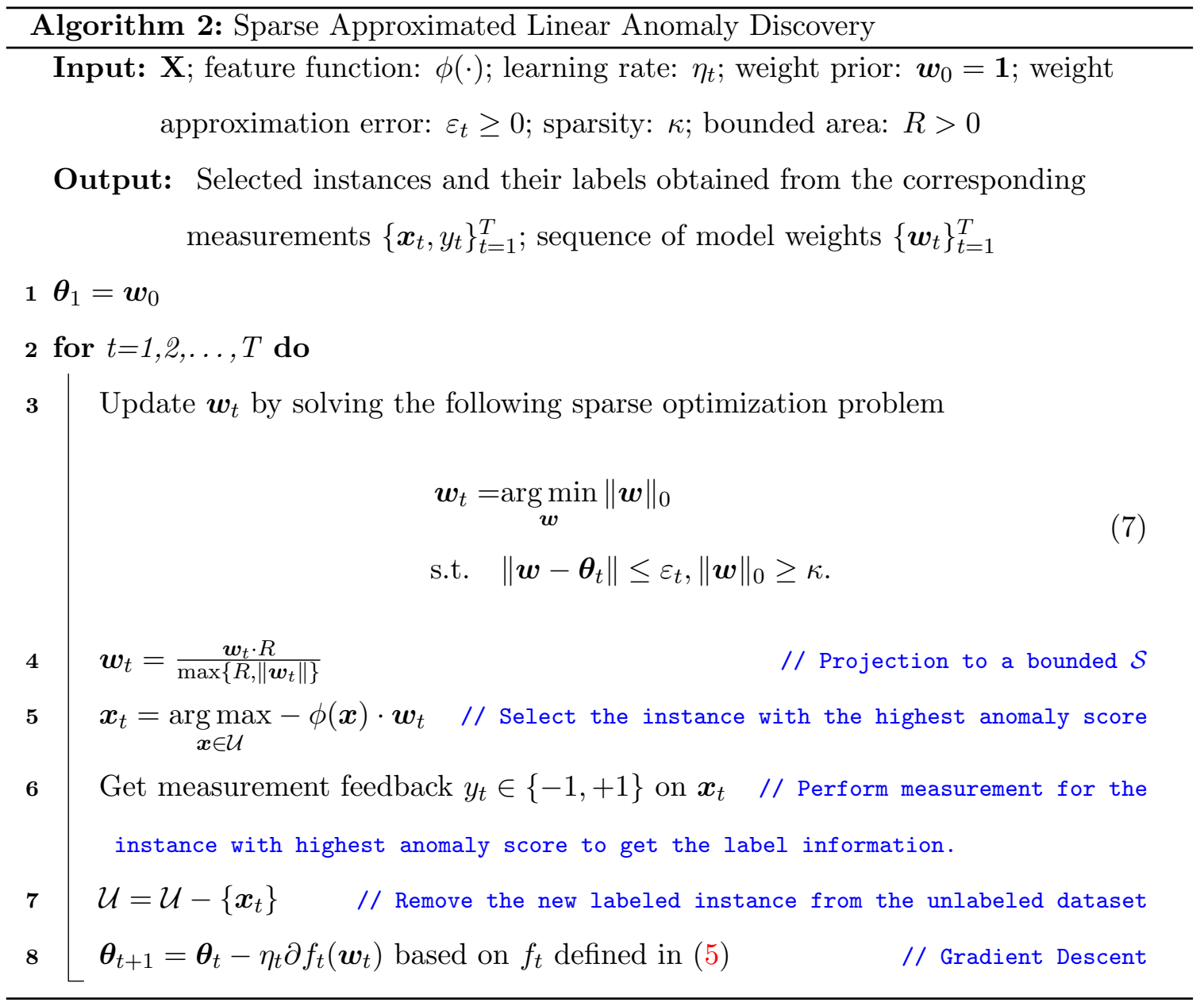

Since $\min _{\boldsymbol{w} \in \mathcal{S}} \sum_{t=1}^{T} f_{t}(\boldsymbol{w})$ is a constant in (6), it can be ignored while minimizing (6). The iterative procedure to minimize (6), where model weight (i.e., decision variable) $\boldsymbol{w}_{t}$ is sequentially obtained from $t=1$ to $T$, can be summarized as follows

$$
\boldsymbol{w}_{1} \rightarrow f_{1}\left(\boldsymbol{w}_{1}\right) \mapsto \boldsymbol{w}_{2} \rightarrow f_{2}\left(\boldsymbol{w}_{2}\right) \mapsto \cdots \mapsto \boldsymbol{w}_{T} \rightarrow f_{T}\left(\boldsymbol{w}_{T}\right)
$$

At each iteration $t, \boldsymbol{w}_{t}$ is determined from the last iteration (represented by $f_{t-1}\left(\boldsymbol{w}_{t-1}\right) \mapsto$ 
$\boldsymbol{w}_{t}$ ), where $f_{t}$ is unseen yet. Once $f_{t}$ is obtained (represented by $\boldsymbol{w}_{t} \rightarrow f_{t}\left(\boldsymbol{w}_{t}\right)$ ), it can be used to update new model weight $\boldsymbol{w}_{t+1}$ (represented by $f_{t}\left(\boldsymbol{w}_{t}\right) \mapsto \boldsymbol{w}_{t+1}$ ). Specifically, it is the standard gradient descent update in Line 8 , where $\boldsymbol{\theta}_{t}$ is an auxiliary variable that assists in weight updating. After $T$ iterations, $\sum_{t=1}^{T} f_{t}\left(\boldsymbol{w}_{t}\right)$ in (6) is achieved. If not considering (7) in Line 3 and setting $\boldsymbol{w}_{t}=\boldsymbol{\theta}_{t}$, algorithm 2 is the standard online gradient descent Streeter and McMahan (2010) to minimize (6). Line 4 is to project $\boldsymbol{w}_{t}$ to a bounded area $\mathcal{S}=\left\{\boldsymbol{w} \in \mathbb{R}^{D}:\|\boldsymbol{w}\| \leq R\right\}$ so that the linear loss is also bounded; otherwise, $\min _{\boldsymbol{w} \in \mathcal{S}} \sum_{t=1}^{T} f_{t}(\boldsymbol{w})$ could be infinity. In Line 5 , based on the current model weight $\boldsymbol{w}_{t}$, the algorithm selects one top-ranked instance $\boldsymbol{x}_{t}$ from the unlabeled dataset, followed by its costly measurement to get the label information $y_{t}$ (Line 6). After selecting $\boldsymbol{x}_{t}$ and receiving $y_{t}$, loss function $f_{t}\left(\boldsymbol{w}_{t}\right)$, which is a part of the objective function in (6), can be obtained through (5). Based on loss function $f_{t}\left(\boldsymbol{w}_{t}\right)$, the $\boldsymbol{\theta}_{t+1}$ is updated in Line 8 through the standard gradient descent update.

In our proposed algorithm, the sparse approximated problem (7) in Line 3 is added to achieve sparsity of $\boldsymbol{w}$ while minimizing (6). It aims to find the most sparse vector $\boldsymbol{w}_{t}$, which is constrained to be close to $\boldsymbol{\theta}_{t}$ and has a reasonable number of nonzero features specified as $\kappa$. The truncation error $\varepsilon_{t}$ in the constraints controls how close between $\boldsymbol{w}_{t}$ and $\boldsymbol{\theta}_{t}$, thus affects the sparsity of $\boldsymbol{w}_{t}$. The parameter $\kappa$ in the constraint of (7) is a small integer, which helps avoid any improper situations where the value of $\varepsilon_{t}$ is set too large. (7) can be efficiently solved to optimal using a simple greedy approach. Specifically, it follows: first, let $\boldsymbol{w}_{t}$ be a copy of $\boldsymbol{\theta}_{t}$; then repeat setting the nonzero element with the smallest absolute value in $\boldsymbol{w}_{t}$ to zero until no more elements can be set to zero without violating the constraints $\left\|\boldsymbol{w}-\boldsymbol{\theta}_{t}\right\| \leq \varepsilon_{t}$ and $\|\boldsymbol{w}\|_{0} \geq \kappa$.

Next, the Regret analysis is discussed, where the Regret is defined in (6) with $\mathcal{S}=\{\boldsymbol{w} \in$ $\left.\mathbb{R}^{D}:\|\boldsymbol{w}\| \leq R\right\}$. The following theorem shows that the Regret of SALAD algorithm 2 .

Theorem 1. SALAD algorithm with learning rate $\left\{\eta_{t}=\frac{\sqrt{2} R}{\sqrt{\sum_{s=1}^{t} \partial f_{t}^{2}\left(\boldsymbol{w}_{t}\right)}}, t \in 1, \ldots, T\right\}$ and approximation error $\left\{\varepsilon_{t}, t \in 1, \ldots, T\right\}$ guarantees the following for all $T \geq 1$

$$
\text { Regret }_{T}=\underbrace{2 \sqrt{2} R \sqrt{\sum_{t=1}^{T}\left\|\phi\left(\boldsymbol{x}_{t}\right)\right\|^{2}}}_{\text {Original Convex Regret }}+\underbrace{\sum_{t=1}^{T} \varepsilon_{t}\left\|\phi\left(\boldsymbol{x}_{t}\right)\right\|}_{\text {Sparse Approximation Regret }}
$$


Proof.

$$
\begin{aligned}
\operatorname{Regret}_{T} & =\sum_{t=1}^{T} f_{t}\left(\boldsymbol{w}_{t}\right)-\min _{\boldsymbol{w} \in \mathcal{S}} \sum_{t=1}^{T} f_{t}(\boldsymbol{w}) \\
& =\sum_{t=1}^{T} f_{t}\left(\boldsymbol{w}_{t}\right)-f_{t}\left(\boldsymbol{\theta}_{t}\right)+\sum_{t=1}^{T} f_{t}\left(\boldsymbol{\theta}_{t}\right)-\min _{\boldsymbol{w} \in \mathcal{S}} \sum_{t=1}^{T} f_{t}(\boldsymbol{w}) \\
& =\sum_{t=1}^{T} y_{t} \phi\left(\boldsymbol{x}_{t}\right) \cdot\left(\boldsymbol{\theta}_{t}-\boldsymbol{w}_{t}\right)+\sum_{t=1}^{T} f_{t}\left(\boldsymbol{\theta}_{t}\right)-\min _{\boldsymbol{w} \in \mathcal{S}} \sum_{t=1}^{T} f_{t}(\boldsymbol{w}) \\
& \leq\left\|\phi\left(\boldsymbol{x}_{t}\right)\right\| \cdot\left\|\boldsymbol{\theta}_{t}-\boldsymbol{w}_{t}\right\|+2 \sqrt{2} R \sqrt{\sum_{t=1}^{T}\left\|\phi\left(\boldsymbol{x}_{t}\right)\right\|^{2}} \\
& \leq\left\|\phi\left(\boldsymbol{x}_{t}\right)\right\| \cdot \varepsilon_{t}+2 \sqrt{2} R \sqrt{\sum_{t=1}^{T}\left\|\phi\left(\boldsymbol{x}_{t}\right)\right\|^{2} .}
\end{aligned}
$$

In the first inequality, $\sum_{t=1}^{T} y_{t} \phi\left(\boldsymbol{x}_{t}\right) \cdot\left(\boldsymbol{\theta}_{t}-\boldsymbol{w}_{t}\right) \leq\left\|\phi\left(\boldsymbol{x}_{t}\right)\right\| \cdot\left\|\boldsymbol{\theta}_{t}-\boldsymbol{w}_{t}\right\|$ is due to the Cauchy inequality and $y_{t}=\{+1,-1\} \cdot \sum_{t=1}^{T} f_{t}\left(\boldsymbol{\theta}_{t}\right)-\min _{\boldsymbol{w} \in \mathcal{S}} \sum_{t=1}^{T} f_{t}(\boldsymbol{w}) \leq 2 \sqrt{2} R \sqrt{\sum_{t=1}^{T}\left\|\phi\left(\boldsymbol{x}_{t}\right)\right\|^{2}}$ is based on the result in Streeter and McMahan (2010); McMahan (2012) and the fact that $\partial f_{t}\left(\boldsymbol{w}_{t}\right)=\partial f_{t}\left(\boldsymbol{\theta}_{t}\right)=-y_{t} \phi\left(\boldsymbol{x}_{t}\right)$. The second inequality comes from the constraint in (7).

The Regret of our proposed SALAD algorithm shown in (8) can be decomposed into two parts. The first part is the original convex Regret, which is the Regret without sparse approximation problem (7). Since $\phi\left(\boldsymbol{x}_{t}\right)$ is proven to be a sparse binary feature in Lemma 1, the original convex Regret has a desirable asymptotic property, namely, no-regret (also called sub-linear convergence rate). That is, $\lim _{T \rightarrow+\infty} \frac{R(T)}{T}=0$. The second part is the sparse approximation Regret, which comes from the sparse approximation problem (7) in Line 3. If $\varepsilon_{t}=\mathcal{O}(1 / \sqrt{T})$, the sparse approximation Regret also has a sub-linear convergence rate. However, when $\varepsilon_{t}$ is set too small, the model weight $\boldsymbol{w}$ is not sparse enough to promote the sparsity in $\phi\left(\boldsymbol{x}_{t}\right)$. When $\varepsilon_{t}$ is set too big, the model weight $\boldsymbol{w}$ is too sparse to generalize well on unseen instances.

Parameter setting of Algorithm 1 and 2 for case studies in Section 4: In Algorithm 1, sub-sample size $\psi$, number of trees $K$, and tree height limit $H$ need to be determined. As suggested in the literature Liu et al. (2008, 2012); Das et al. (2017), $\psi=2^{8}$, $K=100 . \quad H$ is set to a large number so that all instances are isolated in a leaf Siddiqui et al. (2018). In our proposed SALAD Algorithm 2, the learning rate is set to the value in Theorem 1. Based on our empirical results, $\kappa=4,000$ and $\varepsilon_{t}=\sqrt{0.8} \cdot\left\|\boldsymbol{\theta}_{t}\right\|$ are found to be 
very effective in our case studies. $R$ can be set up as any positive value since our anomaly score (2) is a linear function, which is not affected by any scale operation.

\section{Case Studies}

To evaluate the performance of the proposed SALAD, numerical and real-world case studies are conducted in this section. In Section 4.1, the sparsity of feature function $\phi(\cdot)$ and the convergence of SALAD algorithm 2 are investigated with numerically simulated data. In Sections 4.2, 4.3 and 4.4, real-world applications in open-source datasets, a polymer AM dataset, and a metal AM dataset are used to demonstrate the performance of the proposed SALAD. For all analyses in Sections 4.2, 4.3 and 4.4, our proposed SALAD is assessed against the following benchmark methods:

(1) Unsupervised baseline: all instances are sorted in decreasing order of anomaly score (2) computed with the Isolation Forest algorithm given $\boldsymbol{w}=\mathbf{1}$. This algorithm ignores the measurement feedback, and thus the ranking is constant across iterations. This baseline captures the performance of an unsupervised anomaly detection algorithm that does not incorporate measurement feedback.

(2) GLAD: This corresponds to the motivating paper Siddiqui et al. (2018), where the anomaly score is computed using (2) based on Isolation Forest. The algorithm incorporates the measurement feedback by adjusting the model weight $\boldsymbol{w}$ via online mirror descent with non-negative constraints. The instance with the highest anomaly score based on the current model weight is selected to evaluate and get the label at each iteration.

For the evaluation metric, anomaly discovery curve Ding et al. (2019) is utilized to plot the number of discovered anomalies against the number of iterations (measurements). A perfect result is a line with a slope of one, i.e., all the queries are anomalies. The worst case is a line with a slope of zero, i.e., all the queries are normal. All analyses in Sections 4.2, 4.3 and 4.4 are repeated 20 times to get the mean and standard error for comparison. The codes of SALAD are implemented in Matlab 2019a. The CPU of the computer used in the experiments is an Intel ${ }^{\circledR}$ Core $^{\top M}$ Processor i7-6820HQ (Quad Core 2.70 GHz, 3.60 GHz Turbo, $8 \mathrm{MB} 45 \mathrm{~W})$. 


\subsection{Numerical Study}

In this subsection, two cases are considered using simulated data: (a) sparsity analysis of feature function $\phi(\cdot)$ with different sizes of training data $m$; (b) a numerical optimization performance of Algorithm 2 with different $\varepsilon_{t}$.

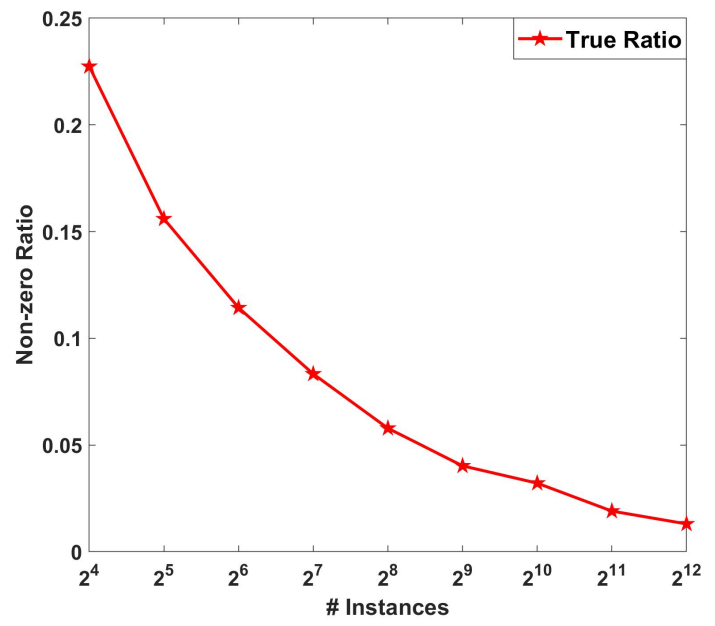

(a) Condition (i): small tree height limit

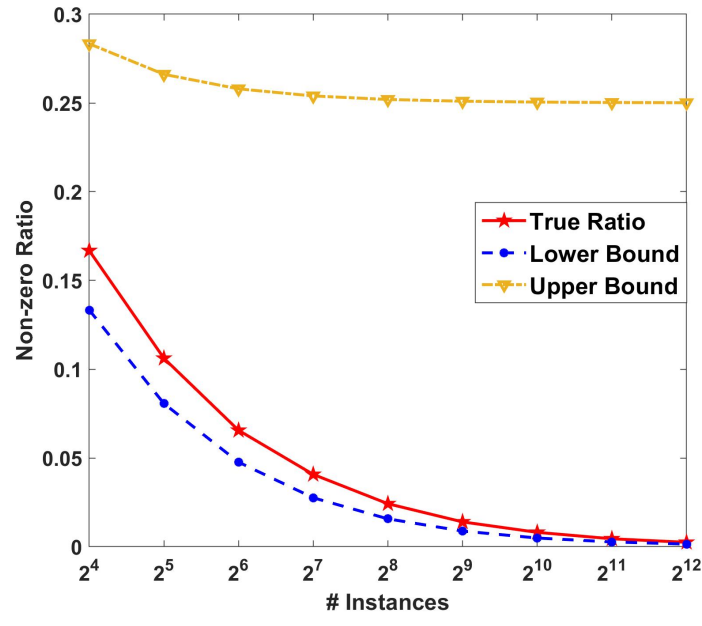

(b) Conditions (ii): large tree height limit

Figure 4: Sparsity analysis of $\phi(\cdot)$ under conditions (i) and (ii) in Lemma 1.

Case (a): The instances used here are simulated independently from $\mathcal{N}(0,5)$. To study the sparsity of feature function $\phi(\cdot)$ as stated in Lemma 1, different numbers of instances are considered for both Conditions (i) and (ii). Specifically, the number of instances can be set to value $m=2^{j}$, where $j=\{4,5, \ldots, 12\}$. For Condition (i), the tree limit height $H$ is set to the value $\lceil\log m\rceil$ as suggested in Liu et al. (2008, 2012). For Condition (ii), the tree limit height $H$ is set to a large number so that all $m$ instances are isolated in a leaf. In Figures $4 \mathrm{a}$ and $4 \mathrm{~b}$, the ratio of nonzero elements is plotted versus the number of instances. The ratio keeps decreasing to zero when the number of instances increases. Furthermore, the lower and upper bounds in (4) are also shown in Figure 4b. It shows that the ratio of nonzero elements is much closer to the lower bound, especially when the number of instances is large.

Case (b): The nominal instances are simulated from $\mathcal{N}(0,5)$ independently, while anomalous instances are simulated from $\mathcal{N}(3,3)$ independently. In total, there are 400 nominal instances and 100 anomalous instances considered in this experiment. To study 
the effect of $\varepsilon_{t}$ in $(7), \varepsilon_{t}$ can take value from $\left\{0,0.2 \cdot\left\|\boldsymbol{\theta}_{t}\right\|, 0.4 \cdot\left\|\boldsymbol{\theta}_{t}\right\|, 0.6 \cdot\left\|\boldsymbol{\theta}_{t}\right\|, 0.8 \cdot\left\|\boldsymbol{\theta}_{t}\right\|\right\}$. If $\varepsilon_{t}=0$, our algorithm degrades to the online gradient descent algorithm used by Streeter and McMahan (2010), which has the sub-linear convergence rate. It can be treated as the oracle for comparison since the optimal solution of the second term on the right-hand side (6) is unknown. In this experiment, Line 5 in Algorithm 2 is replaced by a random policy so that the same instance is used for different $\varepsilon_{t}$ at each iteration. In addition, the sparsity $\kappa$ is set to 4,000 .

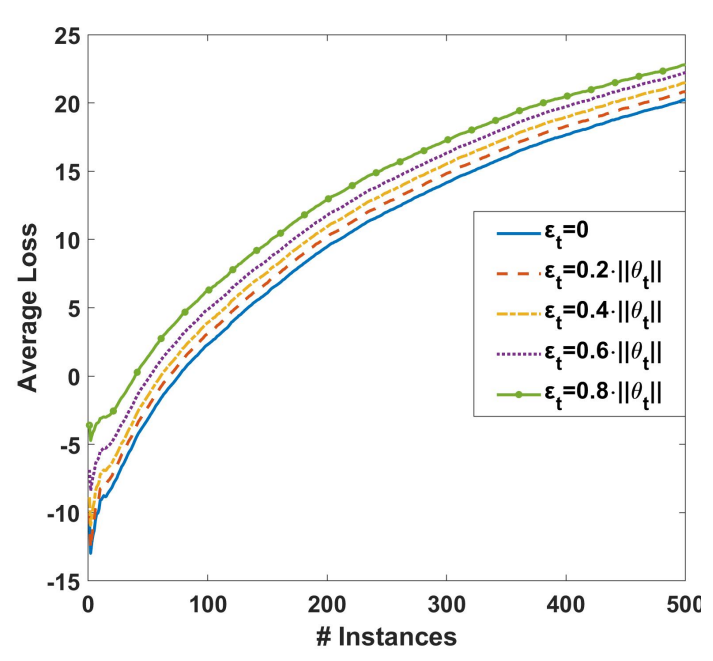

(a) Average loss

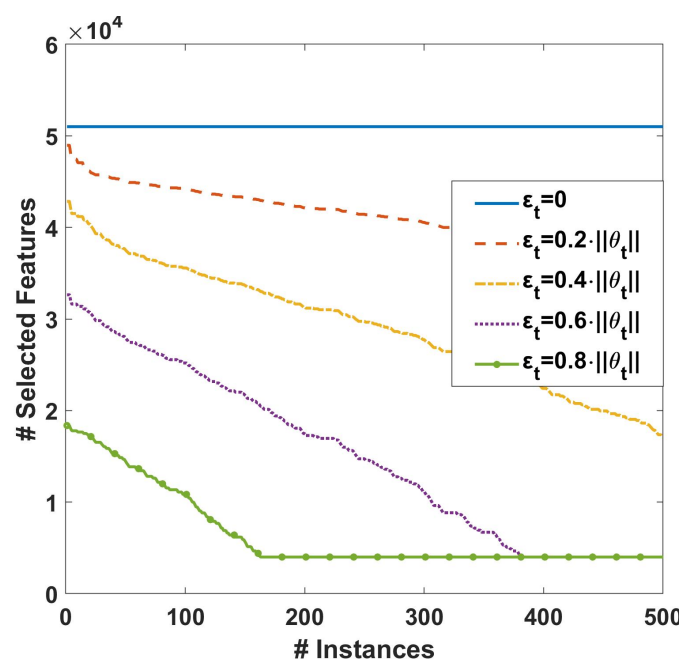

(b) Sparsity of model weight $\boldsymbol{w}_{t}$

Figure 5: Convergence performance of SALAD algorithm (1) Average loss versus number of iterations; (2) sparsity of model weight $\boldsymbol{w}_{t}$ versus number of iterations.

For performance evaluation, the average loss Zhai et al. (2018) defined as $\frac{1}{t} \sum_{i=1}^{t} f_{i}\left(\boldsymbol{w}_{i}\right)$ is plotted versus the number of iterations in Figure $5 \mathrm{a}$, where $f_{i}\left(\boldsymbol{w}_{i}\right)$ is defined in (5). The five curves corresponding to five $\varepsilon$ have a very similar trend. It can be observed that a larger $\varepsilon_{t}$ incurs a larger average loss. It is reasonable since a larger approximation error results in a larger loss. On the other hand, the sparsity of model weight $\boldsymbol{w}_{t}$ is plotted versus the number of iterations for different $\varepsilon_{t}$ in Figure $5 \mathrm{~b}$. For all $\varepsilon_{t}$ except $\varepsilon_{t}=0$, the sparsity keeps decreasing until it reaches $\kappa=4,000$ when the number of iterations increases. Besides, a larger $\varepsilon_{t}$ corresponds to a smaller value of sparsity. 
Table 1: Statistics of Open-source datasets.

\begin{tabular}{|c|c|c|c|c|}
\hline & \# Instances & \# Dim. & \# Anomalies & Anomalies \% \\
\hline Aloi & 50,000 & 27 & 1,508 & 3.02 \\
\hline Annthyroid & 7,200 & 21 & 347 & 4.82 \\
\hline Cardioto & 2,126 & 21 & 86 & 4.05 \\
\hline Heart & 270 & 13 & 7 & 2.59 \\
\hline Kdd & 60,632 & 41 & 200 & 0.33 \\
\hline Letter & 1,600 & 32 & 100 & 6.25 \\
\hline Mammography & 11,183 & 6 & 260 & 2.32 \\
\hline Mnist & 7,603 & 100 & 700 & 9.21 \\
\hline Optdigits & 5,216 & 64 & 150 & 2.88 \\
\hline Pblock & 5,473 & 10 & 99 & 1.81 \\
\hline Process-Miner & 18,398 & 61 & 124 & 0.67 \\
\hline Satellite & 6,435 & 36 & 2,036 & 31.64 \\
\hline Shuttle & 1,013 & 9 & 13 & 1.28 \\
\hline Spambase & 4,601 & 57 & 280 & 6.09 \\
\hline Speech & 3,686 & 400 & 61 & 1.65 \\
\hline Thyroid & 3,772 & 6 & 93 & 2.47 \\
\hline Vowels & 1,456 & 12 & 50 & 3.43 \\
\hline Waveform & 3,443 & 21 & 100 & 2.90 \\
\hline Wilt & 4,839 & 5 & 261 & 5.39 \\
\hline Yeast & 1,364 & 8 & 64 & 4.69 \\
\hline
\end{tabular}

\subsection{Application in Open-source Datasets}

In this subsection, SALAD and benchmark methods are applied to open-source datasets for performance comparison. Twenty datasets selected from Campos et al. (2016); Ranjan et al. (2018) and ODDS ${ }^{4}$ are used in this experiment. The classes were divided into two sets for each dataset, one representing the nominal instances and the other smaller set representing the anomalous instances. The dimension, the number of anomalies, and the percentage

\footnotetext{
${ }^{4}$ http://odds.cs.stonybrook.edu/
} 
Table 2: Results of detected anomalies across 20 runs on open-source datasets for different algorithms.

\begin{tabular}{|c|c|c|c|c|c|c|}
\hline & \multicolumn{2}{|c|}{ Unsupervised } & \multicolumn{2}{|c|}{ GLAD } & \multicolumn{2}{|c|}{ Proposed } \\
\hline & Mean & $\mathrm{SE}$ & Mean & $\mathrm{SE}$ & Mean & $\mathrm{SE}$ \\
\hline Aloi & 27.60 & 3.21 & 57.80 & 6.76 & 122.40 & 7.50 \\
\hline Annthyroid & 71.00 & 9.20 & 300.30 & 13.94 & 333.80 & 11.57 \\
\hline Cardioto & 38.10 & 3.51 & 59.80 & 5.14 & 72.10 & 7.65 \\
\hline Heart & 6.00 & 0.00 & 6.00 & 0.00 & 6.80 & 0.42 \\
\hline Kdd & 102.63 & 3.38 & 137.00 & 5.93 & 145.13 & 5.51 \\
\hline Letter & 32.70 & 3.50 & 31.80 & 4.24 & 72.30 & 5.46 \\
\hline Mammography & 70.90 & 8.44 & 179.80 & 3.77 & 194.50 & 4.72 \\
\hline Mnist & 224.60 & 12.56 & 601.40 & 5.83 & 635.60 & 14.86 \\
\hline Optdigits & 5.70 & 1.64 & 143.50 & 13.57 & 149.90 & 0.32 \\
\hline Pblock & 42.00 & 2.05 & 70.80 & 1.55 & 75.10 & 2.13 \\
\hline Process-Miner & 10.90 & 1.79 & 21.10 & 4.65 & 56.90 & 8.41 \\
\hline Satellite & 1191.70 & 23.82 & 1431.20 & 7.55 & 1708.80 & 17.18 \\
\hline Shuttle & 1.50 & 0.53 & 10.00 & 3.46 & 12.80 & 0.63 \\
\hline Spambase & 119.10 & 6.74 & 217.80 & 3.08 & 247.10 & 3.51 \\
\hline Speech & 4.10 & 1.45 & 4.00 & 1.05 & 5.40 & 1.78 \\
\hline Thyroid & 66.20 & 3.39 & 90.00 & 0.00 & 92.90 & 0.32 \\
\hline Vowels & 14.30 & 2.00 & 39.00 & 1.49 & 48.60 & 1.07 \\
\hline Waveform & 25.10 & 3.51 & 19.00 & 4.69 & 73.20 & 2.49 \\
\hline Wilt & 4.00 & 1.05 & 5.30 & 1.06 & 166.50 & 55.57 \\
\hline Yeast & 29.20 & 1.48 & 32.30 & 0.82 & 34.10 & 0.88 \\
\hline
\end{tabular}

of anomalies in each dataset are summarized in Table 1. For each dataset, the budget $T$ is determined by its number of anomalies. Table 2 shows the quantitative results for all the datasets, including the mean and standard error of the number of detected anomalies. Overall, GLAD and SALAD have much better performance than the unsupervised baseline method, demonstrating the advantage of incorporating measurement feedback in the unsupervised analysis. The proposed SALAD can achieve the best mean performance for 


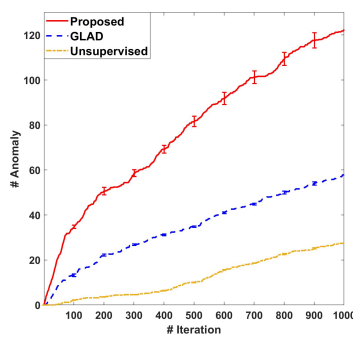

(a) Aloi

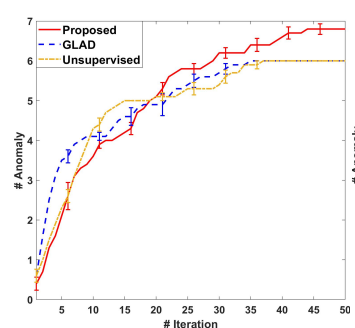

(e) Kdd

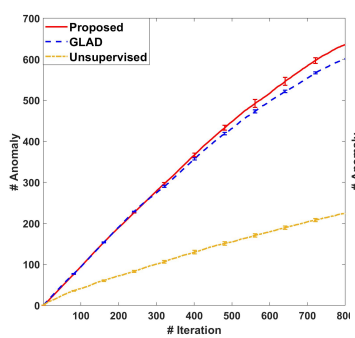

(i) Optdigits

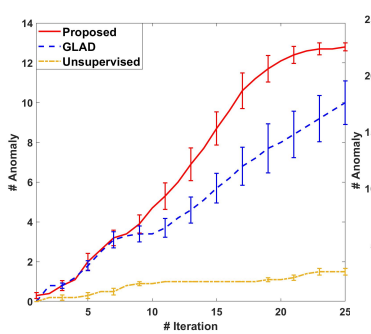

(m) Shuttle

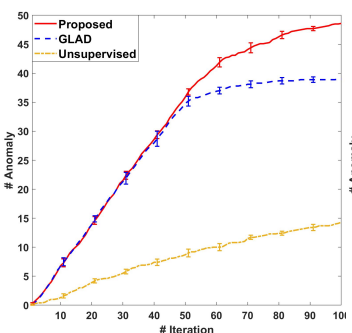

(q) Vowels

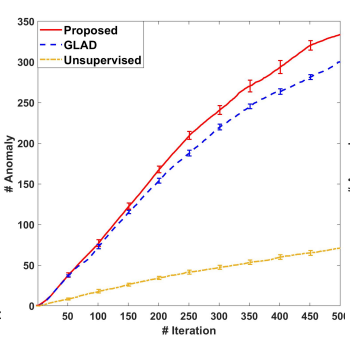

(b) Annthyroid

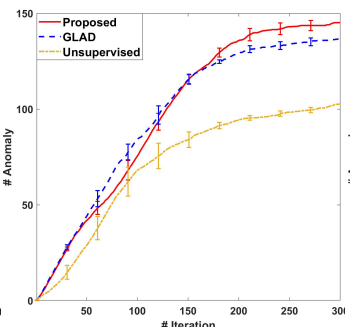

(f) Letter

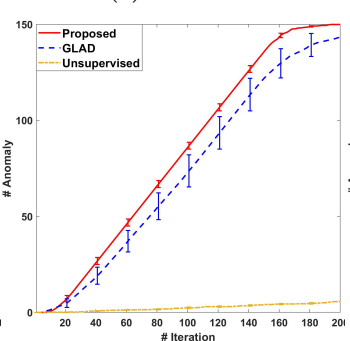

(j) Pblock

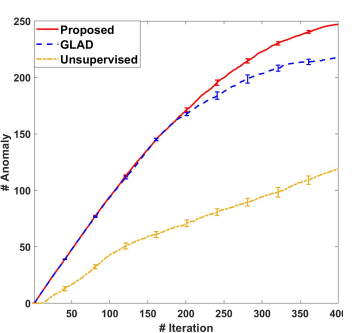

(k) Process-Miner

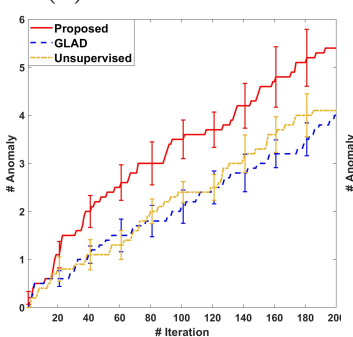

(g) Mammography

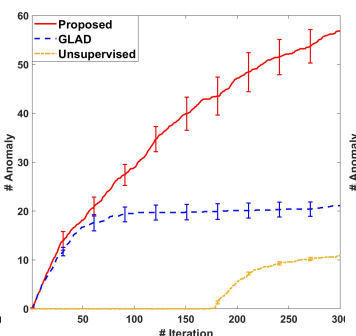

(h) Mnist

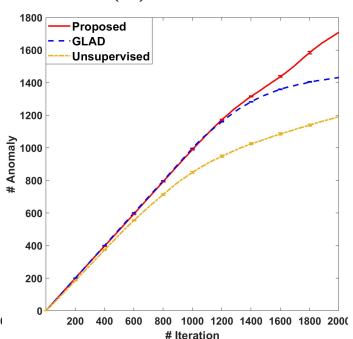

(1) Satellite

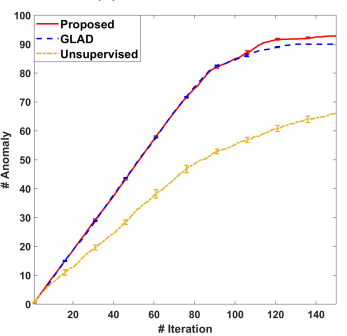

(p) Thyroid

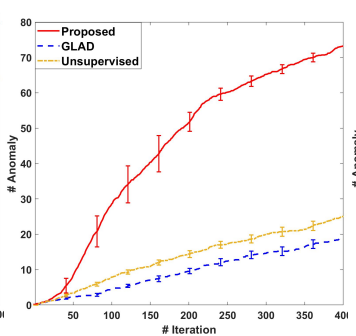

(o) Speech

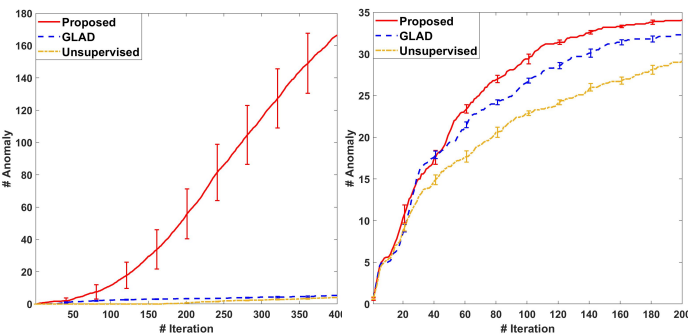

(s) Wilt

(t) Yeast

Figure 6: The total number of detected anomalies versus the number of iterations for all open-source datasets, where the error-bars represent 95\% Confidence Interval. 
all twenty datasets, where it has different levels of improvement over GLAD. On average, the number of anomalies detected by SALAD is 1.97 times more than that detected by GLAD. This illustrates that sparsity can improve the ability to detect anomalies. In terms of standard error, even though the proposed SALAD is not the best, it can generally achieve relatively low standard error.

However, the results in Table 2 only present the performance of different methods when the budget is reached. To explore the performance of different methods during feedback loops, Figure 6 plots the number of detected anomalies versus the number of iterations for all twenty datasets. The curves are averaged over 20 independent runs of the algorithm, and $95 \%$ confidence intervals are also shown. Overall, it illustrates that SALAD never deteriorates the performance of unsupervised baseline (Isolation Forest) and, in most cases, significantly increases the number of anomalies discovered over time compared to both Isolation Forest and GLAD.

\subsection{Application in Polymer Additive Manufacturing}

In this section, a polymer AM dataset Shen et al. (2020) is used to explore the performance of the proposed SALAD on detecting anomalies of interest from an unlabeled dataset. In this experiment, a desktop FFF 3D printer, namely, a Hyrel System 30M 3D printer, is used to print a test artifact, a cuboid of dimensions of 2 inches. In reality, the FFF printing process may encounter defects such as voids, overfill, underfill, etc. However, the root causes of similar defects may be different, leading to the difficulty of defect mitigation. For example, both inappropriate cooling fan settings for temperature control and sub-optimal feed rate (material extrusion speed) may cause underfill, but with different severities. Differentiating these similar defects is critical. Therefore, sensors are installed for online monitoring of the process of printing. High-quality images of the part surface being printed are collected by two digital microscopes at a sampling frequency of $1 \mathrm{~Hz}$, which are installed at the two sides of the extruder of a 3D printer. Images for the surface quality of normal, underfill caused by sub-optimal feed rate, and underfill caused by improper setting of a cooling fan (shown in Figure 7) are collected during the printing, respectively. For each image, a region of interest (ROI) is utilized, which is cropped from the original image $(640 \times 480$ pixels $)$ to a smaller region $(80 \times 80$ pixels $)$ right below the nozzle as shown in Figure 7 . The image in 


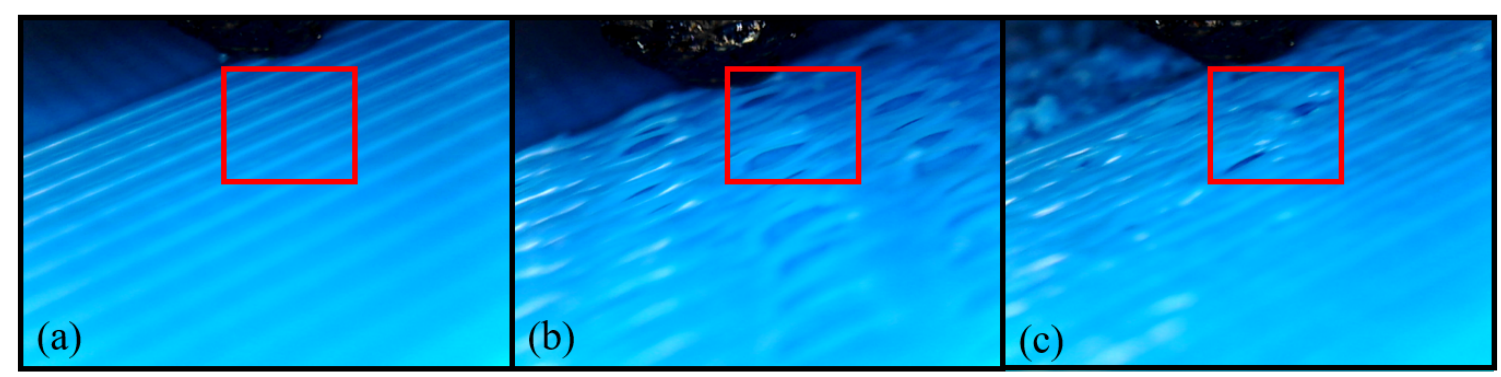

Figure 7: (a) Normal; (b) Underfill caused by improper setting of a cooling fan; (c) Underfill caused by sub-optimal feed rate (red boxes are ROI) Shen et al. (2020).

ROI is transformed into a vector with 6,400 variables for testing different algorithms. For each variable, it represents a pixel value ranging from 0 to 255 . There are 205 images of normal, 153 images of underfill caused by feed rate, and 42 images of underfill caused by the fan, respectively.

The experiment in this subsection has the following procedure.

- Step 1 (Relabeling): 42 images of underfill caused by the fan are labeled as $y=+1$ (defects of interest), while 205 images of normal and 153 images of underfill caused by feed rate are labeled as $y=-1$ (not defects of interest).

- Step 2 (Implementation): All these 400 images in Step 1 are treated as unlabeled images in this analysis. Their actual labels (i.e., $y$ ) are the ground truth that needs to be queried in Line 6 of our proposed algorithm 2. The objective of our algorithm is to detect images of underfill caused by the fan as many as possible under a given budget.

The budget $T$ is set to 100 (the number of measurements). Figure 8 plots the number of detected anomalies versus the number of measurements (iterations). The curves are averaged over 20 independent runs of the algorithm, and $95 \%$ confidence intervals are also shown. The result in Figure 8 implies that the proposed SALAD performs the best since it identifies much more anomalies than the benchmark methods do under the same number of measurements. GLAD improves slightly from the unsupervised baseline. Again, this shows that the advantage of involving sparsity in our proposed algorithm.

In order to gain a deeper understanding of SALAD, the visualization of the polymer AM 


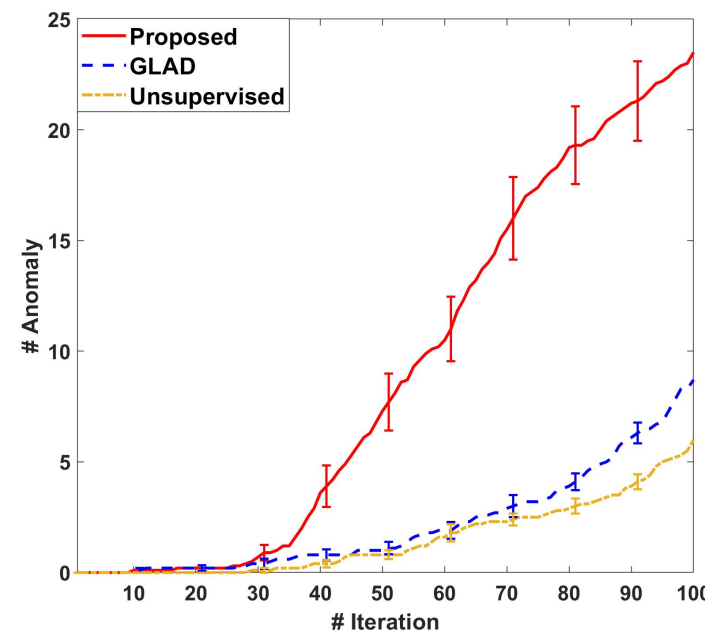

Figure 8: The total number of detected anomalies versus the number of iterations on polymer AM dataset (The error-bars represent 95\% Confidence Interval).

dataset is provided along with the queries by three algorithms. Our intent is to provide some intuitions on how AAD works. For this purpose, t-SNE Van der Maaten and Hinton (2008), a popular dimensionality reduction technique, is applied to produce a two-dimensional (2D) representation of the dataset in this experiment. Figure 9 shows a $2 \mathrm{D}$ representation of the

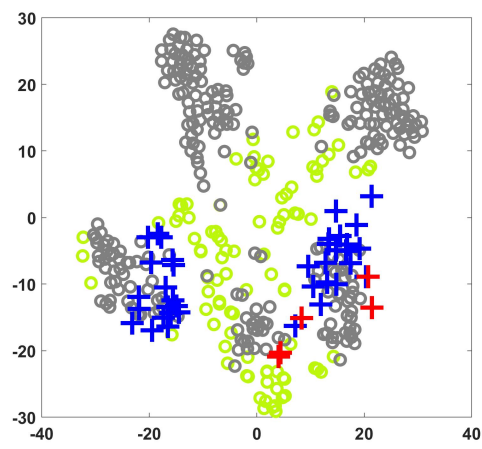

(a) Unsupervised baseline

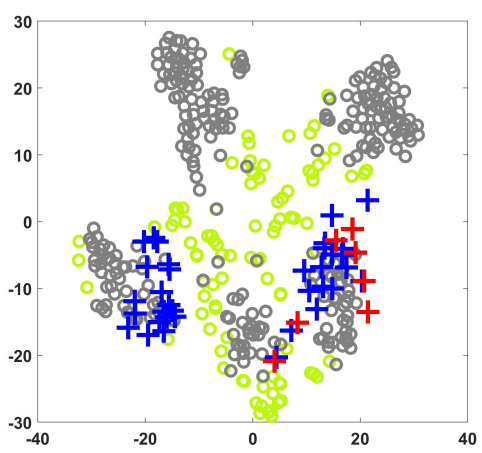

(b) GLAD

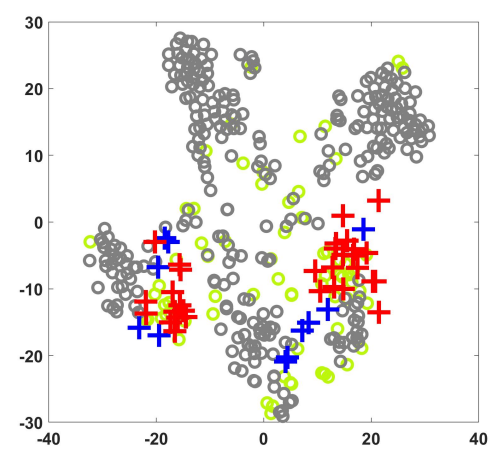

(c) SALAD (Proposed)

Figure 9: Low-dimensional visualization of polymer AM dataset using t-SNE. Plus signs are anomalies, and circles are nominals. A red coloring indicates that a true anomaly point was queried. A green indicates a nominal point was queried. Gray circles correspond to unqueried nominals. To make unqueried anomalies stand out visually, they are indicated with blue plus signs.

polymer AM produced by t-SNE, along with the queries made by benchmarks and SALAD. 
The unsupervised baseline and GLAD tend to discover nominals at the same location shown in the right bottom corner of Figures 9a and 9b. In contrast, our proposed SALAD not only detects more anomalies from the same location but also queries anomalies at more locations as SALAD can detect anomalies shown in the left bottom corner of Figure 9c. Overall, SALAD can query instances more likely to be anomalies and focus on various regions.

\subsection{Application in Metal Additive Manufacturing}

This subsection uses a metal AM dataset from the EBM process Townsend et al. (2016) to evaluate SALAD on the measurement allocation problem. In the printing process, the machine ARCAM Q10 plus is applied to print samples with dimensions $15 \mathrm{~mm} \times 15 \mathrm{~mm} \times$ $25 \mathrm{~mm}$ using Ti-6Al-4V powder. The image shown in Figure 10a is the 2D surface pattern

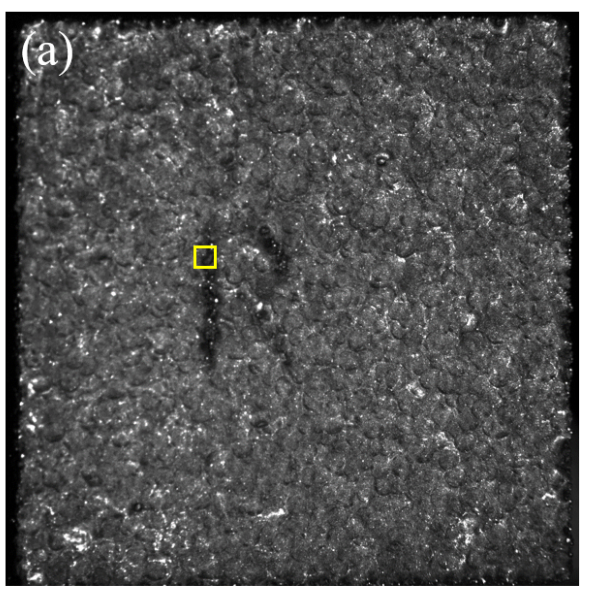

(a) 2D scan image

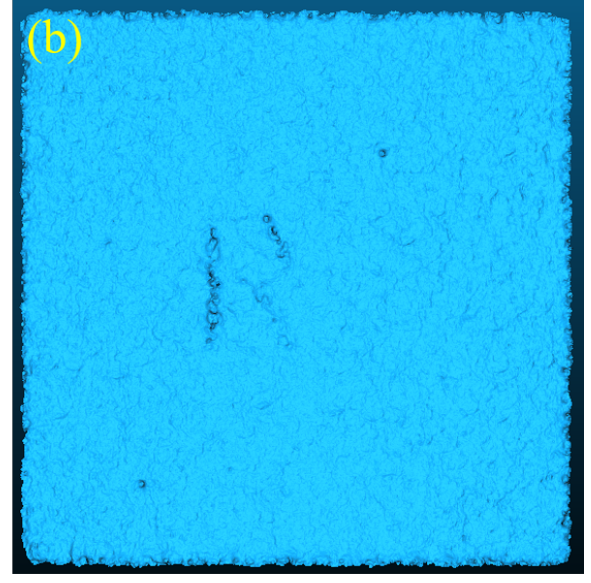

(b) Point cloud data

Figure 10: Metal AM dataset: (a) 2D scan image (yellow square boxes are selected random samples); (b) the corresponding point cloud data.

of the printed part. Surface morphology measurement and quantification can be obtained through a white light interferometer Wang et al. (2021), which has a small field of view (spatial resolution $\sim 20 \mu \mathrm{m}$ ) and takes hours to scan a small surface. The printed part is relatively large $(15 \mathrm{~mm} \times 15 \mathrm{~mm}$ top surface). Therefore, we would like to sample some locations to measure the surface morphology since the measurements are costly, and the cost (the number of measurements) needs to be controlled under the budget. Our proposed algorithm is applied in order to select the locations for Sa (arithmetical mean height of 
an area) Thomas (1981) measurement in order to identify the surface area with inferior surface finishing. To obtain multiple training samples, 200 images with a size of 60 by 60 are randomly selected without overlap from the image in Figure 10a. Each image is transformed into a vector with 3,600 variables, where each variable represents a pixel value ranging from 0 to 255 .

The experiment in this subsection has the following procedure.

- Step 1 (Labeling): For 200 images from Figure 10a, the corresponding surface roughness $\mathrm{Sa}$ is calculated from the point cloud data in the same area, as shown in Figure 10b. The top-7 images that have the highest Sa are labeled as $y=+1$ (anomalies). The remaining images are nominal instances labeled as $y=-1$.

- Step 2 (Implementation): For these 200 images in Step 1, their actual labels (i.e., $y$ ) are the ground truth that needs to be queried in Line 6 of our proposed algorithm 2. The objective of our algorithm is to detect the images that are labeled as $y=+1$ as quick as possible.

The budget $T$ is set to 45 (the number of measurements). Figure 11a plots the number

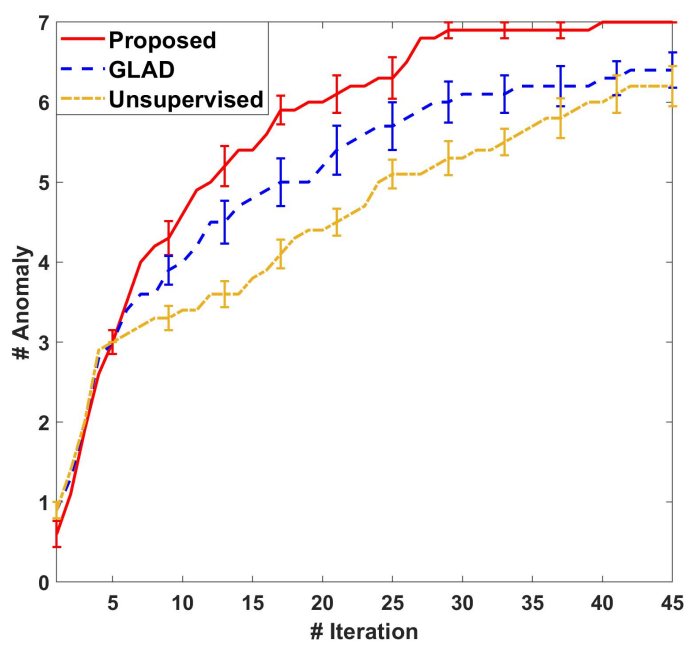

(a)

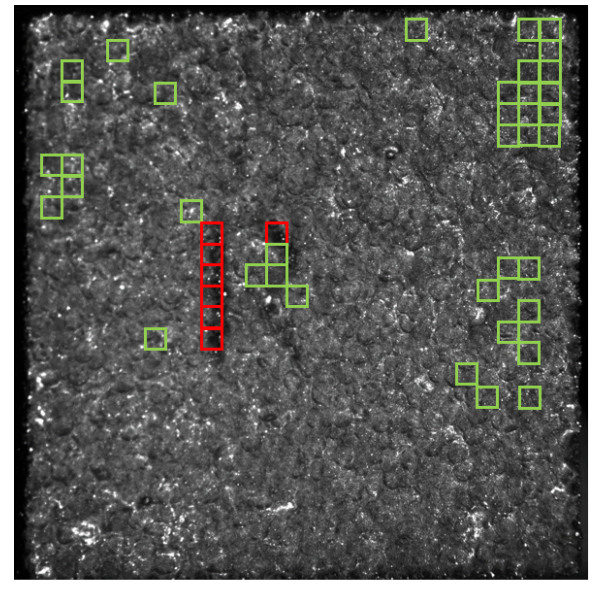

(b)

Figure 11: (a) The total number of detected anomalies versus the number of iterations on metal AM dataset (The error-bars represent 95\% Confidence Interval). (b) Locations of Sa measurements. A red box indicates that a true anomaly point was queried. A green box indicates a nominal point was queried. 
of detected anomalies versus the number of measurements (iterations). The curves are averaged over 20 independent runs of the algorithm, and $95 \%$ confidence intervals are also shown. The result in Figure 11a implies that the proposed SALAD performs better than the unsupervised baseline and GLAD. Specifically, the proposed SALAD detects all seven anomalies within 40 measurements. Figure 11b plots the locations of Sa measurements of our algorithm in the 2D surface view. All anomalies are around the letter "R" in the middle area, which are correctly identified by our algorithm. The other locations of measurements have several clusters, which shows that our algorithm makes accurate decisions on which location to measure with a maximum probability to capture the inferior surface finishing.

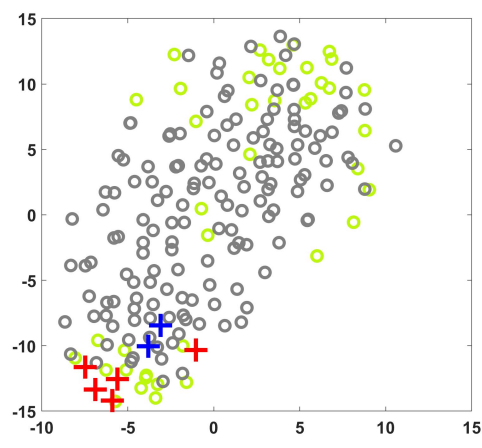

(a) Unsupervised baseline

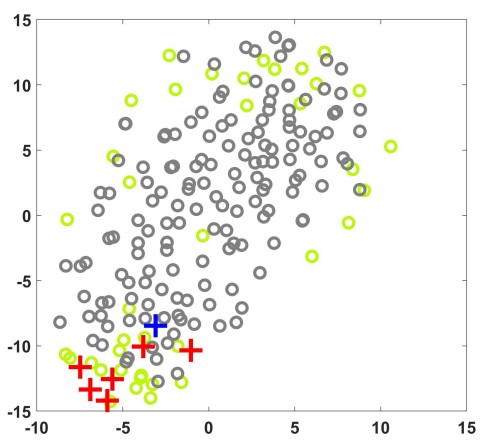

(b) GLAD

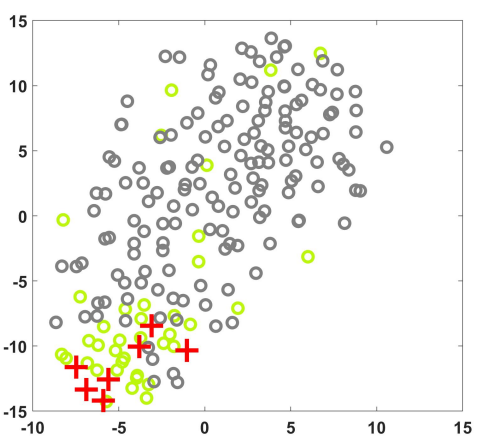

(c) SALAD (Proposed)

Figure 12: Low-dimensional visualization of metal AM dataset using t-SNE. Plus signs are anomalies, and circles are nominals. A red coloring indicates that a true anomaly point was queried. A green indicates a nominal point was queried. Gray circles correspond to unqueried nominals. To make unqueried anomalies stand out visually, they are indicated with blue plus signs.

Same as Section 4.3, the visualization of the metal AM dataset is provided along with the queries by three algorithms. Figure 12 shows a 2D representation of the metal AM produced by t-SNE, along with the queries made by benchmarks and SALAD. The proposed SALAD can detect all seven anomalies in the left bottom corner, as shown in Figure 12c, while the unsupervised baseline and GLAD miss two and one anomaly, respectively. Besides, Figure 12c illustrates that SALAD queries more instances around the anomalous area, demonstrating its effectiveness in anomaly detection. 


\section{Conclusion}

In this article, a new Sparse Approximated Linear Anomaly Discovery algorithm is developed to include sparsity constraint in active anomaly discovery based on isolation forest. The sparsity of feature function from Isolation Forest is studied theoretically and verified empirically to show our motivation to include sparsity. The effectiveness of the proposed algorithm is validated using numerical and real-world case studies. In the numerical study, the empirical convergence shows the tradeoff behavior of the proposed SALAD in terms of regret and model sparsity. Specifically, the proposed SALAD can maintain the model accuracy and sparsity simultaneously. Twenty open-source datasets and two additive manufacturing datasets in real-world applications are applied to evaluate our proposed algorithm. Based on these results, it is evident that SALAD outperforms state-of-the-art algorithms in the literature by including sparsity in the model, especially for datasets with a large number of instances.

In addition, there are still some aspects of SALAD that deserve further investigation. First, the SALAD is a first-order online gradient descent algorithm. The extension to a more advanced online gradient descent algorithm (for example, the second-order OGD Agarwal et al. (2017)) should be further investigated. Second, the query strategy in Line 5 of Algorithm 2 to select the top-1 instance is a greedy approach. The query strategy that can tradeoff exploration and exploitation is one of the next steps of research.

\section{References}

Agarwal, N., Bullins, B., and Hazan, E. (2017). Second-order stochastic optimization for machine learning in linear time. The Journal of Machine Learning Research, 18(1):41484187 .

An, J. and Cho, S. (2015). Variational autoencoder based anomaly detection using reconstruction probability. Special Lecture on IE, 2(1):1-18.

Breunig, M. M., Kriegel, H.-P., Ng, R. T., and Sander, J. (2000). Lof: identifying densitybased local outliers. In Proceedings of the 2000 ACM SIGMOD international conference on Management of data, pages 93-104.

Campos, G. O., Zimek, A., Sander, J., Campello, R. J., Micenková, B., Schubert, E., Assent, I., and Houle, M. E. (2016). On the evaluation of unsupervised outlier detection: measures, datasets, and an empirical study. Data mining and knowledge discovery, 30(4):891-927. 
Chandola, V., Banerjee, A., and Kumar, V. (2009). Anomaly detection: A survey. ACM computing surveys (CSUR), 41(3):1-58.

Das, S., Islam, M. R., Jayakodi, N. K., and Doppa, J. R. (2019). Active anomaly detection via ensembles: Insights, algorithms, and interpretability. arXiv preprint arXiv:1901.08930, 1(1):1-47.

Das, S., Wong, W.-K., Dietterich, T., Fern, A., and Emmott, A. (2020). Discovering anomalies by incorporating feedback from an expert. ACM Transactions on Knowledge Discovery from Data (TKDD), 14(4):1-32.

Das, S., Wong, W.-K., Fern, A., Dietterich, T. G., and Siddiqui, M. A. (2017). Incorporating feedback into tree-based anomaly detection. arXiv preprint arXiv:1708.09441, 1(1):1-9.

Ding, K., Li, J., and Liu, H. (2019). Interactive anomaly detection on attributed networks. In Proceedings of the twelfth ACM international conference on web search and data mining, pages 357-365.

Duchi, J., Shalev-Shwartz, S., Singer, Y., and Chandra, T. (2008). Efficient projections onto the 1 1-ball for learning in high dimensions. In Proceedings of the 25th international conference on Machine learning, pages 272-279.

Hazan, E. (2019). Introduction to online convex optimization. arXiv preprint arXiv:1909.05207, 0(0):1-178.

Kivinen, J., Smola, A. J., and Williamson, R. C. (2004). Online learning with kernels. IEEE transactions on signal processing, 52(8):2165-2176.

Knorr, E. M. and Ng, R. T. (1998). Algorithms for mining distance-based outliers in large datasets. In $V L D B$, volume 98, pages 392-403. Citeseer.

Langford, J., Li, L., and Zhang, T. (2009). Sparse online learning via truncated gradient. Journal of Machine Learning Research, 10(3):777-801.

Liu, C., Law, A. C. C., Roberson, D., and Kong, Z. J. (2019). Image analysis-based closed loop quality control for additive manufacturing with fused filament fabrication. Journal of Manufacturing Systems, 51:75-86.

Liu, F. T., Ting, K. M., and Zhou, Z.-H. (2008). Isolation forest. In 2008 eighth ieee international conference on data mining, pages 413-422. IEEE.

Liu, F. T., Ting, K. M., and Zhou, Z.-H. (2012). Isolation-based anomaly detection. ACM Transactions on Knowledge Discovery from Data (TKDD), 6(1):1-39.

McMahan, B. (2012). Lecture notes in online learning.

Ranjan, C., Reddy, M., Mustonen, M., Paynabar, K., and Pourak, K. (2018). Dataset: rare event classification in multivariate time series. arXiv preprint arXiv:1809.10717, $0(0): 1-7$.

Shalev-Shwartz, S. et al. (2011a). Online learning and online convex optimization. Foundations and trends in Machine Learning, 4(2):107-194. 
Shalev-Shwartz, S., Singer, Y., Srebro, N., and Cotter, A. (2011b). Pegasos: Primal estimated sub-gradient solver for svm. Mathematical programming, 127(1):3-30.

Shen, B., Kamath, R. R., and Hahn Choo, Z. J. K. (2021a). Robust tensor decomposition based background/foreground separation in noisy videos and its applications in additive manufacturing. TechRxiv, 1(1):1-13.

Shen, B., Wang, R., Law, A. C. C., Kamath, R., Choo, H., and Kong, Z. (2021b). Super resolution for multi-sources image stream data using smooth and sparse tensor completion and its applications in data acquisition of additive manufacturing. Technometrics, 1(1):116.

Shen, B., Xie, W., and Kong, Z. J. (2020). Clustered discriminant regression for highdimensional data feature extraction and its applications in healthcare and additive manufacturing. IEEE Transactions on Automation Science and Engineering, 19(19):1-13.

Siddiqui, M. A., Fern, A., Dietterich, T. G., Wright, R., Theriault, A., and Archer, D. W. (2018). Feedback-guided anomaly discovery via online optimization. In Proceedings of the 24th ACM SIGKDD International Conference on Knowledge Discovery 85 Data Mining, pages 2200-2209.

Streeter, M. and McMahan, H. B. (2010). Less regret via online conditioning. arXiv preprint arXiv:1002.4862, 0(0):1-11.

Ten, C.-W., Hong, J., and Liu, C.-C. (2011). Anomaly detection for cybersecurity of the substations. IEEE Transactions on Smart Grid, 2(4):865-873.

Thomas, T. (1981). Characterization of surface roughness. Precision Engineering, 3(2):97104.

Townsend, A., Senin, N., Blunt, L., Leach, R., and Taylor, J. (2016). Surface texture metrology for metal additive manufacturing: a review. Precision Engineering, 46:34-47.

Ukil, A., Bandyoapdhyay, S., Puri, C., and Pal, A. (2016). Iot healthcare analytics: The importance of anomaly detection. In 2016 IEEE 30th international conference on advanced information networking and applications (AINA), pages 994-997. IEEE.

Van der Maaten, L. and Hinton, G. (2008). Visualizing data using t-sne. Journal of machine learning research, 9(11):2579-2605.

Wang, J., Zhao, P., Hoi, S. C., and Jin, R. (2013). Online feature selection and its applications. IEEE Transactions on knowledge and data engineering, 26(3):698-710.

Wang, R., Law, A., Garcia, D., Yang, S., and Kong, Z. (2021). Development of structured light 3d-scanner with high spatial resolution and its applications for additive manufacturing quality assurance. The International Journal of Advanced Manufacturing Technology, $0(0): 1-24$.

Zhai, T., Koriche, F., Wang, H., and Gao, Y. (2018). Tracking sparse linear classifiers. IEEE transactions on neural networks and learning systems, 30(7):2079-2092. 
Zhang, T. (2004). Solving large scale linear prediction problems using stochastic gradient descent algorithms. In Proceedings of the twenty-first international conference on Machine learning, page 116.

Zhao, Y., Nasrullah, Z., and Li, Z. (2019). Pyod: A python toolbox for scalable outlier detection. Journal of Machine Learning Research, 20(96):1-7. 